\title{
Inexact Picard iterative scheme for steady-state nonlinear diffusion in random heterogeneous media
}

\author{
P. Surya Mohan, Prasanth B. Nair, ${ }^{*}$ and Andy J. Keane \\ Computational Engineering and Design Group. School of Engineering Sciences, University of Southampton, Highfield. \\ Southmapton SOI7 1BJ United Kingdom \\ (Received 10 November 2008; published 13 April 2009)
}

\begin{abstract}
In this paper, we present a numerical scheme for the analysis of steady-state nonlinear diffusion in random heterogeneous media. The key idea is to iteratively solve the nonlinear stochastic governing equations via an inexact Picard iteration scheme, wherein the nonlinear constitutive law is linearized using the current guess of the solution. The linearized stochastic governing equations are then spatially discretized and approximately solved using stochastic reduced basis projection schemes. The approximation to the solution process thus obtained is used as the guess for the next iteration. This iterative procedure is repeated until an appropriate convergence criterion is met. Detailed numerical studies are presented for diffusion in a square domain for varying degrees of nonlinearity. The numerical results are compared against benchmark Monte Carlo simulations, and it is shown that the proposed approach provides good approximations for the response statistics at modest computational effort.
\end{abstract}

DOI: 10.1103/PhysRevE.79.046706

PACS number(s): 02.30.Jr, 02.30.Mv, 02.50.Fz, 02.70.Dh

\section{INTRODUCTION}

Steady-state nonlinear diffusion equations are an important class of partial differential equations (PDEs) which arise from the mathematical characterization of a variety of diffusion phenomena appearing in nature. Numerical simulations of such systems are often carried out in a deterministic setting, even though it is recognized that diffusion parameters and boundary conditions are fraught with considerable randomness. It is now becoming increasingly standard practice to numerically solve stochastic PDE (SPDE) models of diffusion that employ probabilistic models of uncertain parameters in order to rationally quantify variability in predictions. The problem of steady-state diffusion in random heterogeneous media has been studied by many researchers (see, for example, $[1-10])$ in the past for its diverse applications in several areas including heat transfer, petroleum reservoir characterization, and water resources modeling.

A number of researchers have applied Monte Carlo simulation methods to study diffusion in random heterogeneous media. Even though Monte Carlo techniques are very general purpose in scope, the associated computational cost becomes prohibitive for problems where a fine spatial mesh is required to resolve the solution characteristics. More efficient alternatives based on sparse quadrature rules have been employed in $[3,4,11,12]$. This approach leverages the regularity of stochastic variables in contrast to Monte Carlo and quasiMonte Carlo schemes. Perturbation methods have also been widely applied to this problem, but the results are known to become inaccurate for high degree of variability in the input parameters; see, for example, [8-10] and references therein. Tartakovsky et al. $[8,9]$ considered nonlinear flow in random heterogeneous media, where the nonlinear SPDEs are linearized via the Kirchoff mapping and the response statistics are subsequently approximated using a first-order perturbation

*Corresponding author: p.b.nair@soton.ac.uk method. Note that a special form of nonlinearity was considered which admits linearization using Kirchoff mapping and more general forms of nonlinearity do not necessarily admit such a linearization step [13]. There is also a wide body of literature focusing on stochastic diffusion problems where white-noise models of diffusivity and boundary conditions are employed $[14,15]$. In the present work, we focus on stochastic diffusion problems where the diffusivity is modeled by a random field with finite variance (i.e., a second-order stochastic process).

Projection schemes based on polynomial chaos (PC) expansions have been employed in $[1,5,6]$. In this approach, the SPDE solution is expanded in a PC basis with undetermined spatially varying coefficients. Galerkin projection is used to derive a system of coupled deterministic PDEs for the undetermined coefficients that are subsequently solved using a standard numerical PDE solution technique such as finite element or finite difference methods. For problems with many random variables, this approach can be computationally expensive since Galerkin projection typically results in a large system of coupled PDEs. The generalized spectral decomposition approach was recently proposed in the literature $[16,17]$ to circumvent this computational issue. Stochastic reduced basis methods (SRBMs) were proposed in $[18,19]$ to enable the efficient solution of linear SPDEs by seeking a solution in a preconditioned stochastic Krylov subspace. Numerical studies have shown that SRBMs offer considerable computational gains compared to $\mathrm{PC}$ projection schemes for solving linear elliptic SPDEs [20]. Considering that, while solving a nonlinear SPDE iteratively (irrespective of the iterative scheme employed), a linear SPDE has to be solved at each step, this advantage can also be leveraged with nonlinear SPDEs.

In the present paper, we focus on the nonlinear stochastic steady-state diffusion equation, where the diffusivity is parametrized in terms of a random field with finite memory. We employ the Picard iteration scheme to linearize the governing stochastic equations which are subsequently spatially dis- 
cretized using a finite element formulation. The linear random algebraic system of equations arising at each Picard iteration is then solved in an inexact sense using SRBMs. While the focus of the present work is on a model nonlinear diffusion equation, it is worth noting that our approach is general in scope and can be applied to a wider class of elliptic SPDEs.

We present detailed numerical studies for a model SPDE governing diffusion in random heterogeneous media with nonlinear diffusivity. The results obtained using the proposed inexact stochastic Picard iterative scheme are compared against those obtained using a standard Monte Carlo simulation procedure. It is shown that the proposed approach provides good approximations at a fraction of the computational effort required for a high-resolution Monte Carlo simulation.

The remainder of this paper is organized as follows. In Sec. II, we present some preliminary theoretical materials and describe the model nonlinear diffusion equation studied in the present work. The detailed formulation of the proposed numerical scheme is discussed in Sec. III. The computational and implementation aspects of the proposed approach are discussed in Sec. IV. In Sec. V, we present a numerical study on the diffusion equation with stochastic diffusivity. Here we compare the convergence trends of our numerical scheme for cases where the diffusivity is modeled as either linear or (weakly or strongly) nonlinear. We conclude the paper in Sec. VI and highlight some directions for future research.

\section{PROBLEM DEFINITION}

In this section we present the problem definition and prove a theoretical result establishing the existence of a fixed point for the nonlinear SPDE under consideration. Let $(\Omega, \mathcal{F}, \mathcal{P})$ be a probability space, where $\Omega$ is the sample space, $\mathcal{F}$ is the $\sigma$ algebra associated with $\Omega$, and $\mathcal{P}$ is a probability measure. By definition, $L_{2}(\Omega, \mathcal{F}, \mathcal{P})$ is a Hilbert space of random variables. The inner product between two random vectors $\mathbf{u}(\xi)$ and $\mathbf{v}(\xi)$ is defined as

$$
(\mathbf{u}(\xi), \mathbf{v}(\xi))=\left\langle\mathbf{u}^{*}(\xi) \mathbf{v}(\xi)\right\rangle=\int \mathbf{u}^{*}(\xi) \mathbf{v}(\xi) d \mathcal{P}(\xi),
$$

where $\xi$ is a random variable in $L_{2}(\Omega, \mathcal{F}, \mathcal{P})$, the superscript ${ }^{*}$ denotes the complex-conjugate transpose, and $\langle\cdot\rangle$ denotes the expectation operator.

Consider the following nonlinear stochastic steady-state diffusion equation:

$$
\begin{gathered}
\nabla \cdot[\kappa(x, u, \omega) \nabla u(x, \omega)]=f(x, \omega) \quad \text { in } D \times \Omega, \\
\mathcal{B} u(x, \omega)=g(x, \omega) \text { on } \partial D \times \Omega,
\end{gathered}
$$

where $f(x, \omega)$ is a random field defined on $D \times \Omega$ and $g(x, \omega)$ is a random field defined on $\partial D \times \Omega . D \subset \mathbb{R}^{\ell}, \ell=1,2,3$, and $\partial D$ denotes the physical domain and its boundary, respectively. $\mathcal{B}$ is an operator indicating the type of boundary conditions that are imposed, e.g., Dirichlet, Neumann, or mixed boundary conditions. $u(x, \omega)$ is the field variable whose spatial statistics are sought to be computed.

Following Matthies and Keese [3,4], we model the nonlinear stochastic diffusivity field $\kappa(x, u, \omega)$ as

$$
\kappa(x, u, \omega)=\hat{\kappa}(x, \omega)+\lambda u^{2}(x, \omega),
$$

where $\hat{\kappa}(x, \omega)$ is a random field and $\lambda$ is a parameter governing the degree of nonlinearity.

Before delving into the numerical algorithms for solving Eq. (1), it is important to investigate if a solution (fixed point) indeed exists for the SPDE. In order to theoretically prove the existence of the fixed point for Eq. (1), we need to introduce the following function spaces.

$L_{p}(\Omega)$ space: Let $1 \leq p \leq \infty$ and $(\Omega, \mathcal{F}, \mathcal{P})$ be as above. Then the $L_{p}(\Omega)$ space is the set of all measurable functions from $\Omega$ to $\mathbb{R}$ whose absolute value raised to the $p$ th power has a finite Lebesgue integral or

$$
\|f\|_{p}=\left(\int \mid f^{p} d \mathcal{P}\right)^{1 / p}<\infty .
$$

$W_{p}^{k}(D)$ : For $p \geq 1, W_{p}^{k}(D)$ refers to the set of all functions $u \in L_{p}(D)$ such that all derivatives of $u$ of orders lesser than or equal to $k$ belong to $L_{p}(D)$.

Now we investigate the appropriate function spaces to define $u$ and $f$ for the well posedness of problem (1). In contrast to the case where the diffusivity is linear, we need to define the spatial part in a Sobolev space $W_{p}^{1}(D)$ with $p>2$. Hence $u(x, \omega) \in W_{p}^{1}(D) \times L_{p}(\Omega)$. On the other hand $f(x, \omega)$ should be in the dual space of $W_{p}^{1}(D) \times L_{p}(\Omega)$, namely, $f$ $\in W^{*}(D) \times L_{q}(\Omega)$ where $W^{*}(D)=W_{q}^{-1}(D)$ and $q$ is related to $p$ as $1 / p+1 / q=1$.

Since the nonlinearity in Eq. (2) is quadratic in nature, if $p=4$, we can define a continuous nonlinear operator [21] on $W_{p}^{1}(D) \times L_{p}(\Omega)$ as follows:

$\mathcal{N}=\left[\hat{\kappa}(x, \omega)+\lambda u^{2}(x, \omega)\right] \nabla u(x, \omega), \quad \forall u \in W_{p}^{1}(D) \times L_{p}(\Omega)$.

Define a semilinear (linear in $v$ ) form on $W_{p}^{1}(D) \times L_{p}(\Omega)$ as follows:

$$
a(u, v)=\left\langle\int_{D} \nabla v \cdot \mathcal{N}(u) \mathrm{d} x\right\rangle, \quad \forall u, v \in W_{p}^{1}(D) \times L_{p}(\Omega) .
$$

Now consider the variational formulation given by

$$
a(u, v)=L(v), \quad \forall u, v \in W_{p}^{1}(D) \times L_{p}(\Omega),
$$

where the linear form $L$ is defined as

$$
L(v)=\left\langle\int_{D} f v d x\right\rangle
$$

The variational formulation (3) has a unique solution for all $f \in W^{*}(D) \times L_{q}(\Omega)$ by the well-known Lax-Milgram lemma [22] provided that $\mathcal{N}(u)>0$. We ensure the positivity of $\mathcal{N}(u)$ by considering the diffusivity to be bounded from below and above, i.e.,

$$
0<\kappa_{\min }<\kappa(x, u, \omega)<\kappa_{\max }<\infty .
$$

The stochastic linear component of the diffusivity, i.e., the term $\hat{\kappa}(x, \omega)$ in Eq. (2), can be ensured to be positive valued by choosing an appropriate random-field model (e.g., lognormal random field). The term $u^{2}(x, \omega)$ in the diffusivity model (2) is always positive valued everywhere. 
To justify the application of the Picard iteration scheme, we first state that the following Dirichlet problem:

$$
\begin{gathered}
\nabla \cdot[\kappa(x, u, \omega) \nabla u(x, \omega)]=f(x, \omega) \text { in } D \times \Omega, \\
u(x, \omega)=g(x, \omega) \text { on } \partial D \times \Omega,
\end{gathered}
$$

with the nonlinearity model (2) and the assumptions listed above has a unique fixed point. The interested reader is referred to the Appendix for a proof of this result.

Theorem: for a given $v \in W_{p}^{1}(D) \times L_{p}(\Omega)$, define an operator $T(\omega)$ by letting $u=T v$ be the unique solution in $W_{p}^{2}(D)$ $\times L_{p}(\Omega)$ of the linear Dirichlet problem,

$$
\begin{gathered}
\nabla \cdot[\kappa(x, v, \omega) \nabla u(x, \omega)]=f(x, \omega) \text { in } D \times \Omega, \\
u(x, \omega)=g(x, \omega) \text { on } \partial D \times \Omega,
\end{gathered}
$$

where $f \in W^{*}(D) \times L_{q}(\Omega)$ and $g \in W_{p}^{\mathrm{l}}(\partial D) \times L_{p}(\Omega)$. Then $T(\omega)$ has a fixed point.

We model the linear component of the diffusivity by a lognormal random field, which can be defined by a transformation of a Gaussian random field $Y(x, \omega)$ as

$$
\hat{\kappa}(x, \omega)=\exp [Y(x, \omega)] .
$$

Karhunen-Loève expansion $[4,23,24]$ of a lognormal random field results in a set of random variables whose probabilistic structure cannot be easily determined. Ghanem [25] proposed to discretize the underlying Gaussian random field $Y(x, \omega)$ using the Karhunen-Loève expansion scheme and then expand the lognormal field in a Hermite PC basis. Using this approach, the discretized lognormal random field can be expressed in the form

$$
\hat{\kappa}(x, \omega) \approx \sum_{i=0}^{P_{1}} \hat{\kappa}_{i}(x) \phi_{i}(\xi)
$$

Here we use the symbol $\xi$ to denote the vector of uncorrelated random variables arising from Karhunen-Loève discretization of the random fields $Y, f$, and $g,{ }^{1}$ and $\left\{\phi_{i}\right\}$ denotes a set of Hermite PC basis functions. For simplicity of notation, we shall henceforth assume that $\hat{\kappa}(x, \omega), f(x, \omega)$, and $g(x, \omega)$ are statistically independent. We wish to point out that this assumption is primarily made for notational convenience and to improve clarity of presentation.

Since we assumed that $f(x, \omega)$ is a random field on $W^{*}$ $\times L_{q}(\Omega)$, it can also be represented in the general Hermite $\mathrm{PC}$ expansion form ${ }^{2}$

$$
f(x, \omega) \approx \sum_{i=0}^{P_{f}} f_{i}(x) \phi_{i}(\xi)
$$

\footnotetext{
${ }^{1}$ Strictly speaking, the random variables appearing in Eq. (6) are those arising from Karhunen-Loève expansion of $Y(x, \omega)$ alone. However, we group together all random variables arising from random-field discretization in a single vector for notational convenience.

${ }^{2}$ Note that the above representation in a Hermite PC basis is optimal only when $f(x, \omega)$ is a Gaussian random field. For more general distributions, a specially constructed set of orthogonal polynomials may be necessary to ensure optimal convergence [26].
}

Before moving on to the spatial discretization of the model nonlinear SPDE described in this section, we first review the application of generalized SRBMs to solve linear random algebraic systems of equations.

\section{STOCHASTIC REDUCED BASIS METHODS}

SRBMs $[18,19,27,28]$ can be applied to solve linear random algebraic systems of equations of the form

$$
\mathbf{K}(\boldsymbol{\xi}) \mathbf{u}(\xi)=\mathbf{f}(\xi),
$$

where $\mathbf{K}(\boldsymbol{\xi})$ and $\mathbf{f}(\boldsymbol{\xi})$ are expressed in a PC basis as

$$
\mathbf{K}(\boldsymbol{\xi})=\sum_{i=0}^{P} \mathbf{K}_{i} \phi_{i}(\boldsymbol{\xi}) \text { and } \mathbf{f}(\boldsymbol{\xi})=\sum_{i=0}^{P} \mathbf{f}_{i} \phi_{i}(\boldsymbol{\xi})
$$

The underlying idea of SRBMs is to approximate the solution vector $\mathbf{u}(\xi)$ in Eq. (8) using basis vectors spanning the preconditioned stochastic Krylov subspace defined as

$$
\mathcal{K}_{m}(\overline{\mathbf{K}(\boldsymbol{\xi})}, \widetilde{\mathbf{f}(\boldsymbol{\xi})})=\operatorname{span}\left\{\widetilde{\mathbf{f}(\boldsymbol{\xi})}, \widetilde{\mathbf{K}(\boldsymbol{\xi})} \widetilde{\mathbf{f}(\xi)}, \ldots, \widetilde{\mathbf{K}(\xi)^{m-1}} \widetilde{\mathbf{f}(\xi)}\right\},
$$

where $\overline{\mathbf{K}(\boldsymbol{\xi})}=\mathbf{M K}(\boldsymbol{\xi}), \widehat{\mathbf{f}(\boldsymbol{\xi})}=\mathbf{M f}(\boldsymbol{\xi})$, and $\mathbf{M} \in \mathbb{R}^{n \times n}$ is a deterministic left preconditioner. In previous studies using SRBMs $[18,27,28]$, the matrix $\mathbf{K}_{0}^{-1}$ was chosen as the preconditioner and this choice has been shown to provide good convergence. However, here we present a more general derivation which allows the specification of alternative preconditioners that may be required in situations which necessitate $h$ refinement in the random space to ensure high accuracy [29].

\section{A. Basis vector representation}

The basis vectors spanning the preconditioned stochastic Krylov subspace, $\mathcal{K}_{m}(\overrightarrow{\mathbf{K}(\xi)}, \overline{\mathbf{f}(\xi)})$, can be recursively computed as $\psi_{0}(\boldsymbol{\xi})=\widehat{\mathbf{f}(\boldsymbol{\xi})}$ and $\psi_{p+1}(\boldsymbol{\xi})=\overline{\mathbf{K}(\xi)} \psi_{p}(\boldsymbol{\xi})$, where $1 \leq p$ $\leq m-1$. The basis vectors $\left\{\boldsymbol{\psi}_{0}(\boldsymbol{\xi}), \boldsymbol{\psi}_{1}(\boldsymbol{\xi}), \ldots, \boldsymbol{\psi}_{m}(\boldsymbol{\xi})\right\}$ are second-order stochastic processes and hence admit meansquare convergent representation in a PC basis as a result of the Cameron-Martin theorem [30]. Hence, the basis vector $\psi_{p}(\xi)$ for any $p \geq 0$ can be approximated as

$$
\psi_{p}(\xi)=\sum_{i=0}^{P_{u}} \psi_{i}^{p} \phi_{i}(\xi)
$$

Using Eq. (9), the PC expansion coefficients of $\psi_{0}(\xi)$ $=\mathbf{M f}(\xi)$ can be written as

$$
\begin{gathered}
\psi_{k}^{0}=\mathbf{M f}_{k}, \quad \forall 0 \leq k \leq P, \\
\psi_{k}^{0}=0, \quad k>P .
\end{gathered}
$$

Since the $(p+1)$ th basis vector can be written as $\psi_{p+1}(\boldsymbol{\xi})=\mathbf{M K}(\xi) \psi_{p}(\xi)$ for $p \geq 0$, we have

$$
\boldsymbol{\psi}_{p+1}(\boldsymbol{\xi})=\sum_{i=0}^{P} \sum_{j=0}^{P_{u}} \mathbf{M K}_{i} \boldsymbol{\psi}_{j}^{p} \phi_{i} \phi_{j}=\sum_{k=0}^{P_{u}} \boldsymbol{\psi}_{k}^{p+1} \phi_{k} .
$$

The undetermined coefficients in the PC expansion, $\psi_{k}^{p+1}$, can be obtained by projecting them onto the $\mathrm{PC}$ basis, which leads to the following expression: 


$$
\begin{gathered}
\psi_{k}^{p+1}=\frac{\sum_{i=0}^{P} \sum_{j=0}^{P_{u}} \mathbf{M K}_{i} \psi_{j}^{p}\left\langle\phi_{i} \phi_{j} \phi_{k}\right\rangle}{\left\langle\phi_{k}^{2}\right\rangle}, \\
\forall 0 \leq k \leq P_{u} \quad \text { and } \quad p \geq 0 .
\end{gathered}
$$

Due to the PC expansion of each basis vector, the set of basis vectors that approximately span the preconditioned stochastic Krylov subspace $\boldsymbol{\Psi}(\xi)=\left\{\boldsymbol{\psi}_{0}(\xi)\right.$, $\left.\psi_{1}(\xi), \psi_{2}(\xi), \ldots, \psi_{m}(\xi)\right\}$ can be compactly rewritten in matrix notation as follows:

$$
\boldsymbol{\Psi}(\xi)=\sum_{i=0}^{P_{u}} \boldsymbol{\Gamma}_{i} \phi_{i}
$$

where $\boldsymbol{\Gamma}_{i}=\left[\boldsymbol{\psi}_{i}^{0}, \boldsymbol{\psi}_{i}^{1}, \boldsymbol{\psi}_{i}^{2}, \ldots, \boldsymbol{\psi}_{i}^{n}\right] \in \mathbb{R}^{n \times(m+1)}$ is a deterministic matrix.

Thus the stochastic reduced basis representation of the response vector can be written as

$$
\mathbf{u}(\xi)=\Psi(\xi) \alpha=\left(\sum_{i=0}^{P_{u}} \Gamma_{i} \phi_{i}\right) \boldsymbol{\alpha},
$$

where $\boldsymbol{\alpha}^{T}=\left\{\alpha_{0}, \alpha_{1}, \alpha_{2}, \ldots, \alpha_{m}\right\}$ is a vector of undetermined deterministic coefficients which can be computed using Galerkin projection.

\section{B. Stochastic Galerkin projection}

Substituting Eqs. (9) and (10) into the linear random algebraic system of Eqs. (8), we have

$$
\left[\sum_{i=0}^{P} \mathbf{K}_{i} \phi_{i}(\boldsymbol{\xi})\right] \hat{\boldsymbol{\Psi}}(\boldsymbol{\xi}) \boldsymbol{\alpha}=\sum_{i=0}^{P} \mathbf{f}_{i} \phi_{i}(\boldsymbol{\xi}) .
$$

The residual vector resulting from the stochastic reduced basis approximation can be written as

$$
\boldsymbol{\epsilon}(\boldsymbol{\xi})=\left[\sum_{i=0}^{P} \mathbf{K}_{i} \phi_{i}(\xi)\right] \hat{\boldsymbol{\Psi}}(\xi) \alpha-\sum_{i=0}^{P} \mathbf{f}_{i} \phi_{i}(\xi) .
$$

In order to compute the undetermined coefficients $\boldsymbol{\alpha}$, we apply a Galerkin projection scheme where the residual is made orthogonal to the basis vectors $\left\{\hat{\psi}_{i}(\xi)\right\}$, i.e., $\boldsymbol{\epsilon}(\boldsymbol{\xi}) \perp \hat{\boldsymbol{\psi}}_{i}(\boldsymbol{\xi})$, where $i=1,2, \ldots, m+1$. Imposing orthogonality between the stochastic residual and the approximating space of basis vectors in the $L_{2}$ sense results in the following $(m+1) \times(m+1)$ reduced-order system of deterministic equations:

$$
\left(\sum_{i=0}^{P_{u}} \sum_{j=0}^{P} \sum_{k=0}^{P_{u}} \boldsymbol{\Gamma}_{i}^{T} \mathbf{K}_{j} \boldsymbol{\Gamma}_{k}\left\langle\phi_{i} \phi_{j} \phi_{k}\right\rangle\right) \boldsymbol{\alpha}=\sum_{i=0}^{P^{\prime}} \boldsymbol{\Gamma}_{i}^{T} \mathbf{f}_{i}\left\langle\phi_{i}^{2}\right\rangle,
$$

where $P^{\prime}=\min \left(P, P_{u}\right)$. Typically $m \ll n$ where $n$ is the number of degrees of freedom in the system. Thus the preceding equations can be efficiently solved for the undetermined coefficients $\boldsymbol{\alpha}$. It is worth noting here that Eq. (12) is equivalent to a reduced-order model of the equations arising from the
Ghanem-Spanos PC projection scheme [23] that is constructed using a set of deterministic basis vectors spanning a block diagonal preconditioned Krylov subspace.

Note that the response process (10) can be alternatively written as

$$
\mathbf{u}(\xi)=\sum_{i=0}^{P_{u}} \mathbf{u}_{i} \phi_{i}, \quad \text { where } \mathbf{u}_{i}=\Gamma_{i} \boldsymbol{\alpha} .
$$

\section{PICARD ITERATION SCHEME}

In this section, we present an inexact Picard iteration scheme that employs SRBMs for solving the model nonlinear SPDE presented in Sec. $\Pi$. Recall that the linear component of diffusivity $\hat{\kappa}(x, \omega)$ in Eq. (2) has been represented in a PC basis in Eq. (6). We now discuss linearization of the nonlinear component so that the overall diffusivity is in a PC basis which in turn facilitates spatial discretization of the governing equations.

We employ the Picard iteration scheme to linearize the nonlinear governing equations. Let $u_{0}$ be an initial guess of the solution which is assumed to be expanded in a PC basis without loss of generality as follows ${ }^{3}$ :

$$
u_{0}(x, \xi)=\sum_{i=0}^{P_{0}} u_{0_{i}}(x) \phi_{i}(\xi)
$$

The nonlinear diffusivity in Eq. (2) is linearized with $u_{0}$ and then the governing Eq. (1) is spatially discretized. The linear algebraic systems of equations that arise are solved using generalized SRBMs presented in Sec. III. The approximation to the response thus obtained [which is in a PC basis-see Eq. (13)] is then used to linearize the governing equations. These equations are spatially discretized and this iterative cycle is repeated until convergence.

Let the solution at any iterative step be written as $v$. Then the next iterate $u$ is computed by solving the following linearized equation:

$$
\begin{gathered}
\nabla \cdot[\kappa(x, v, \omega) \nabla u(x, \omega)]=f(x, \omega) \quad \text { in } D \times \Omega, \\
\mathcal{B} u(x, \omega)=g(x, \omega) \text { on } \partial D \times \Omega .
\end{gathered}
$$

It can be seen that the structure of Eq. (14) is similar to the linear SPDE considered in [29]. Hence SRBMs can be directly applied to approximate $u$.

In brief, the continuum equations are spatially discretized using finite element approximations resulting in a linear random algebraic system of equations represented in a PC basis. The solution of this system of equations is approximated using basis vectors spanning the preconditioned stochastic Krylov subspace, and the current guess is updated to the response obtained and substituted in Eq. (14). These steps of updating the current guess and uncertainty propagation are

\footnotetext{
${ }^{3} u_{0}$ is stochastic if an approximate solution to the nonlinear system is known or it can be deterministic, in which case only $u_{0_{0}}$ is nonzero.
} 
repeated until an appropriate stopping criterion is met.

In Secs. IV A-IV C that follow, we outline the steps involved in spatial discretization of the linearized governing equations, computing the stochastic reduced basis approximation at each iteration, the stopping criterion, and the postprocessing steps.

\section{A. Spatial discretization}

A finite element spatial discretization is assumed where the spatial domain $D \subset \mathbb{R}^{3}$ is divided into subdomains using a finite element mesh. Consider a typical finite element $D^{e}$ with shape functions $\left\{N_{i}^{e}(x)\right\} \in W^{h} \subset W$ defined over its nodes. Here $W^{h}$ is a finite element space with the maximum element diameter $h>0$. Then the vector representation of the finite element approximation of the solution over the element $D^{e}$ can be written as

$$
u^{e}(x, \boldsymbol{\xi})=\mathbf{N}^{e}(x) \mathbf{u}^{e}(\boldsymbol{\xi}) .
$$

Similarly, from the previous iteration, we have the finite element approximation of the current guess solution $v$ over the same discretized spatial domain. Substituting these finite element approximations into the weak form of the governing equations yields the following expressions for the element matrices:

$$
\begin{gathered}
\mathbf{K}^{e}(\boldsymbol{\xi})=\int_{D^{e}} \nabla \mathbf{N}^{e}(x)\left[\hat{\kappa}(x, \boldsymbol{\xi})+\lambda v^{2}(x, \boldsymbol{\xi})\right]\left(\nabla \mathbf{N}^{e}(x)\right)^{T} d x \\
\mathbf{f}^{e}(\boldsymbol{\xi})=\int_{D^{e}} f(x, \boldsymbol{\xi})\left(\mathbf{N}^{e}(x)\right)^{T} d x
\end{gathered}
$$

From Eqs. (7) and (16), the element force vector can be written as

$$
\begin{aligned}
\mathbf{f}^{e}(\boldsymbol{\xi}) & =\int_{D^{e}} \sum_{i=0}^{P_{f}} f_{i}(x)\left(\mathbf{N}^{e}(x)\right)^{T} \phi_{i}(\boldsymbol{\xi}) d x \\
& =\sum_{i=0}^{P_{f}}\left[\int_{D^{e}} f_{i}(x)\left(\mathbf{N}^{e}(x)\right)^{T} d x\right] \phi_{i}(\boldsymbol{\xi}),
\end{aligned}
$$

where $f_{i}(x)$ can be approximated over the element $D^{e}$ (using centroid, ${ }^{4}$ shape function, spatial average, or other approximations) and the element force vector can be computed using a quadrature rule (e.g., the Gauss quadrature scheme) of appropriate order. Recall that $\hat{\kappa}(x, \xi)$ is already available in a PC expansion [refer to Eq. (6)]. It can be readily seen from Eq. (16) that once we represent the nonlinear term $v^{2}(x, \xi)$ in a PC basis, the element stiffness matrix can be readily computed.

From the previous iterative step, we have a stochastic reduced basis approximation of the current guess response $v(x, \boldsymbol{\xi})$ in form (13), i.e.,

\footnotetext{
${ }^{4}$ The value of $f_{i}(x)$ over an element is approximated by its value at the centroid of the element.
}

$$
v(x, \boldsymbol{\xi})=\sum_{i=0}^{P_{v}} v_{i}(x) \phi_{i}(\boldsymbol{\xi})
$$

With $v(x, \boldsymbol{\xi})$ expressed in a PC basis, the term $v^{2}(x, \boldsymbol{\xi})$ can also be readily written in a PC basis as

$$
v^{2}(x, \boldsymbol{\xi})=\sum_{i=0}^{P_{v^{2}}} \eta_{i}(x) \phi_{i}(\boldsymbol{\xi}),
$$

where $\eta_{k}(x)=\left\langle v^{2}(x, \xi) \phi_{k}\right\rangle /\left\langle\phi_{k}^{2}\right\rangle$. Hence from Eqs. (6) and (19) the diffusivity can be written as

$$
\begin{aligned}
\kappa(x, v(x, \boldsymbol{\xi}), \boldsymbol{\xi}) & =\hat{\kappa}(x, \boldsymbol{\xi})+\lambda v^{2}(x, \boldsymbol{\xi}) \\
& =\sum_{i=0}^{P_{k}}\left[\hat{\kappa}_{i}(x)+\lambda \eta_{i}(x)\right] \phi_{i}(\boldsymbol{\xi}),
\end{aligned}
$$

where $P_{k}=\max \left(P_{1}, P_{v^{2}}\right)$. Now substituting Eq. (20) into the expression for the element stiffness matrix (16) leads to

$$
\mathbf{K}^{e}(\xi)=\sum_{i=0}^{P_{k}} \mathbf{K}_{i}^{e} \phi_{i}(\xi)
$$

where

$$
\mathbf{K}_{i}^{e}=\int_{D^{e}} \nabla \mathbf{N}^{e}(x)\left[\hat{\kappa}_{i}(x)+\lambda \eta_{i}(x)\right]\left(\nabla \mathbf{N}^{e}(x)\right)^{T} d x .
$$

The structure of Eqs. (17) and (21) is similar to equations for the element matrices that arise when spatially discretizing linear SPDEs. The functions $\left[f_{i}(x), \hat{\kappa}_{i}(x)\right.$, and $\left.\eta_{i}(x)\right]$ can be approximated over each element using various approximation techniques, for example, centroid, spatial average, or shape function based approximations. Assembly of the element matrices and incorporation of appropriate boundary conditions thus lead to a linear random algebraic system of equations of form (8), where the global stiffness and force matrices are given by Eq. (9) [here $\left.P \geq \max \left(P_{k}, P_{f}\right)\right]$. The resulting Eq. (8) is iteratively solved to approximate the response statistics.

\section{B. Convergence criterion and numerical issues}

We terminate the iterations when the $L_{2}$ norm of the difference between the consecutive solutions $\mathbf{v}(\xi)$ and $\mathbf{u}(\xi)$ is less than or equal to the user specified tolerance $\epsilon_{\mathrm{tol}}$, i.e.,

$$
\|\mathbf{u}(\boldsymbol{\xi})-\mathbf{v}(\boldsymbol{\xi})\|<\epsilon_{\mathrm{tol}} \text {. }
$$

The $L_{2}$ norm in the preceding equation can be simplified (using orthogonality of PC basis functions) as

$$
\begin{aligned}
\|\mathbf{u}(\xi)-\mathbf{v}(\xi)\| & =\left\langle[\mathbf{u}(\boldsymbol{\xi})-\mathbf{v}(\boldsymbol{\xi})]^{T}[\mathbf{u}(\boldsymbol{\xi})-\mathbf{v}(\boldsymbol{\xi})]\right\rangle^{1 / 2} \\
& =\left[\sum_{i=1}^{P^{\prime}}\left(\mathbf{u}_{i}-\mathbf{v}_{i}\right)^{T}\left(\mathbf{u}_{i}-\mathbf{v}_{i}\right)\left\langle\phi_{i}^{2}\right\rangle\right]^{1 / 2},
\end{aligned}
$$

where $P^{\prime} \geq \max \left(P_{u}, P_{v}\right)$.

In Eq. (8), note that both the current guess and updated solutions, namely, $\mathbf{v}(\boldsymbol{\xi})$ and $\mathbf{u}(\boldsymbol{\xi})$, are expanded using 
SRBMs (and PC basis). Ideally, $\mathbf{u}(\boldsymbol{\xi})$ should be expanded in a higher-order SRBM basis (and hence a higher-order PC basis) compared to $\mathbf{v}(\boldsymbol{\xi})$ for numerical stability. But increasing the order of the basis at each iterative step is not computationally feasible. Hence the basis is truncated in realworld applications which may adversely affect the stability of the iterative scheme in certain cases. This problem can be alleviated by using an adaptive approach wherein the number of stochastic basis vectors is chosen based on the residual norm [which can be readily estimated, for example, using the approximation given in Eq. (23)] in the numerical approximation of Eq. (8). Alternatively, $h$-refinement strategies [29] can be employed to refine the approximation locally in the probability space so that lower-order global basis vectors can provide better accuracy. We shall later demonstrate numerically in Sec. V that the inexact Picard iteration scheme converges satisfactorily for the model problem considered for various strengths of nonlinearity.

\section{Postprocessing}

In this section, we derive the first two statistical moments of the response process. Higher-order moments can be computed similarly. The mean of the response vector is the expectation of $\mathbf{u}(\boldsymbol{\xi})$ given by Eq. (13), i.e.,

$$
\boldsymbol{\mu}_{u}=\langle\mathbf{u}(\boldsymbol{\xi})\rangle=\left\langle\left(\sum_{i=0}^{P_{u}} \mathbf{u}_{i} \phi_{i}\right)\right\rangle=\mathbf{u}_{0}
$$

where the RHS is as a result of the property of PC basis functions, $\left\langle\phi_{i}\right\rangle=0, \forall i>0$ [23]. The covariance of $\mathbf{u}(\xi)$ is given by

$$
\mathbf{C}_{u}=\left\langle\left(\mathbf{u}-\boldsymbol{\mu}_{u}\right)\left(\mathbf{u}-\boldsymbol{\mu}_{u}\right)^{T}\right\rangle .
$$

After some algebraic manipulations, the covariance matrix of the solution vector can be compactly written as

$$
\mathbf{C}_{u}=\sum_{i=0}^{P_{u}} \mathbf{u}_{i} \mathbf{u}_{i}^{T}\left\langle\phi_{i}^{2}\right\rangle-\mathbf{u}_{0} \mathbf{u}_{0}^{T} .
$$

We now show how to estimate the $L_{2}$ norm of the residual $\epsilon(\xi)$ in Eq. (8). In practice, it would be useful to monitor the convergence of the full nonlinear residual at each iteration. This, however, will involve the use of sampling schemes that would be computationally expensive. The $L_{2}$ norm of the residual error of the linear subproblem solved at each Picard iteration can be computed as

$$
\|\boldsymbol{\epsilon}(\boldsymbol{\xi})\|_{2}^{2}=\sum_{i=0}^{P_{\xi}} \boldsymbol{\epsilon}_{i}^{T} \boldsymbol{\epsilon}_{i}\left\langle\phi_{i}^{2}\right\rangle
$$

where

$$
\boldsymbol{\epsilon}_{k}=\frac{\sum_{i=0}^{P} \sum_{j=0}^{P_{u}} \mathbf{K}_{i} \mathbf{u}_{j}\left\langle\phi_{i} \phi_{j} \phi_{k}\right\rangle-\mathbf{f}_{k}\left\langle\phi_{k}^{2}\right\rangle}{\left\langle\phi_{k}^{2}\right\rangle},
$$

and $P_{\epsilon} \geq \max \left(P, P_{u}\right)$ is the order of the PC expansion used for representing the residual vector. In our numerical studies, we use the $L_{2}$ norm of the residual and the true error norm computed via Monte Carlo simulation to analyze the convergence trends of SRBMs.

\section{Treating other types of nonlinearities}

In Secs. IV A-IV C, we focused on the additive type of nonlinearities. SPDEs with more general forms of nonlinearities can also be tackled using the proposed numerical scheme. For example, consider a multiplicative uncertainty model $[8,9,13]$ where the diffusivity is written in the form

$$
\kappa(x, u, \boldsymbol{\xi})=\hat{\kappa}(x, \boldsymbol{\xi}) u(x, \boldsymbol{\xi}) .
$$

Although the above nonlinearity can be linearized by transforming the field variable using the Kirchoff transformation $[8,9]$, it can be alternatively treated using the proposed stochastic Picard iterative scheme. Given a PC basis representation of both the linear random diffusivity and the response process as Eqs. (6) and (18), the nonlinear diffusivity can be expanded in a higher-order PC basis as follows:

$$
\begin{gathered}
\kappa(x, u, \xi)=\sum_{k=0}^{P^{\prime}} \kappa_{k}(x) \phi_{k}, \\
\kappa_{k}(x)=\sum_{i=0}^{P_{1}} \sum_{j=0}^{P_{u}} \hat{\kappa}_{i}(x) u_{j}(x) \frac{\left\langle\phi_{i} \phi_{j} \phi_{k}\right\rangle}{\left\langle\phi_{k}^{2}\right\rangle},
\end{gathered}
$$

where $P^{\prime} \geq \max \left(P_{1}, P_{u}\right)$.

The above equation when substituted into the variational formulation gives rise to a linearized system of equations from where the procedure follows according to the steps described in Secs. IV A-IV C. We wish to highlight here that the present approach can be readily applied to a wide range of nonlinear stochastic diffusion problems provided that we have an accurate PC representation of the constitutive laws.

\section{NUMERICAL STUDIES}

Consider a square domain defined by $D=[0,1]^{2}$ with homogeneous Neumann boundary conditions on the walls $(x=0, x=1)$ and Dirichlet boundary conditions $u=0$ and $u$ $=1$ on the lower and upper boundaries, respectively. The stochastic diffusivity given by Eq. (2) is nonlinear on the domain with no sources or sinks. The linear stochastic component of the diffusivity given by $\hat{k}(x, \omega)$ is modeled as a statistically homogeneous lognormal random field with an isotropic exponential covariance function,

$$
C_{Y}(r)=\sigma_{Y}^{2} \exp (-r / b),
$$

where $r=|\mathbf{x}-\mathbf{y}|$ and $b$ is the correlation length. In all the numerical studies we have set the correlation length $b=0.2$ and the mean of the lognormal random field $\langle\hat{k}(x, \omega)\rangle=1$ and variance $\sigma_{Y}^{2}=1$. The parameter that controls the degree of nonlinearity, namely, $\lambda$ in Eq. (2) is set to $\lambda=0,0.1,0.5$, and 1 to represent linear, weak, moderate, and strong nonlinear diffusivities, respectively. A finite element spatial discretization was performed using a mesh of triangular elements with 2763 degrees of freedom and 5528 elements. 

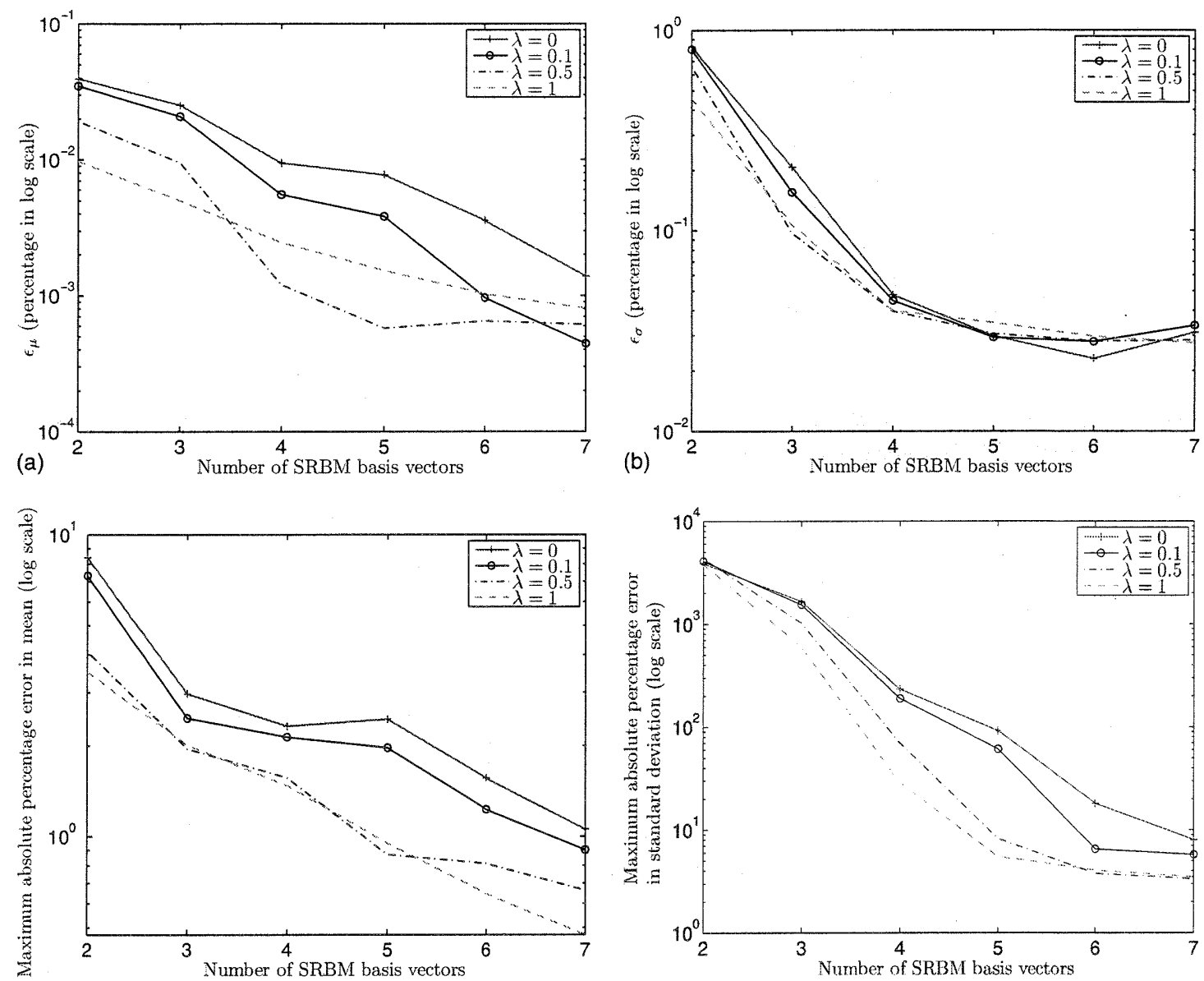

(c)

(d)

FIG. 1. (Color online) Convergence metrics: (a) $\epsilon_{\mu}$, (b) $\epsilon_{\sigma}$, (c) maximum percentage error in mean, and (d) maximum percentage error in standard deviation for different strengths of nonlinearities.

A Monte Carlo simulation with 100000 samples is first conducted to generate a set of benchmark results against which the performance of the inexact stochastic Picard iteration scheme is compared. The underlying Gaussian random field $Y(x, \omega)$ of the lognormal model for the linear diffusivity term $\hat{\kappa}(x, \omega)$ is discretized using the Karhunen-Loève expansion scheme [23] and ten random variables are retained in the expansion. $\hat{\kappa}(x, \xi)$ is then expanded in a second-order Hermite PC basis in terms of ten Gaussian random variables. For all the numerical studies, we start with an initial guess of zero and the iterations are terminated when the norm given in Eq. (22) is less than or equal to $10^{-6}$. The Monte Carlo mean and standard deviation are computed from the solutions of these simulations. Note that errors involved in these simulations are only due to truncated representation of $\hat{\kappa}(x, \omega)$ and not due to the truncation of PC expansions of the response process.

We now present some error metrics computed by comparing the results generated by Monte-Carol simulation (MCS) and the proposed numerical approach that employs the $\mathrm{Pi}$ card iterative scheme in conjunction with SRBMs. We restrict the expansion of $\hat{\kappa}(x, \xi)$ to a second-order PC basis in terms of ten Gaussian random variables for all the strengths of nonlinearities $(\lambda)$. The initial guess is considered to be zero over the entire spatial domain and the iterations are terminated when the $L_{2}$ norm of the difference between successive solutions is less than or equal to $10^{-6}$. Convergence is studied for a varying number of SRBM basis vectors where each stochastic basis vector is truncated to a secondorder PC expansion. For each case, the inexact Picard scheme takes around 12 iterations to meet the stopping criterion in Eq. (22) with $\epsilon_{\mathrm{tol}}=10^{-6}$.

Figure 1 shows four error metrics, namely, relative norm errors and maximum errors in mean and standard deviation, respectively. The relative norm error in the mean and standard deviation are defined as follows:

$$
\begin{gathered}
\epsilon_{\mu}=\frac{\left\|\mu_{\mathrm{MCS}}-\mu_{\mathrm{SRBM}}\right\|}{\left\|\mu_{\mathrm{MCS}}\right\|}, \\
\epsilon_{\sigma}=\frac{\left\|\sigma_{\mathrm{MCS}}-\sigma_{\mathrm{SRBM}}\right\|}{\left\|\sigma_{\mathrm{MCS}}\right\|} .
\end{gathered}
$$

This figure shows that the numerical scheme provides good approximations for the response statistics as the number of basis vectors is increased. The linear problem $(\lambda=0)$ is simi- 


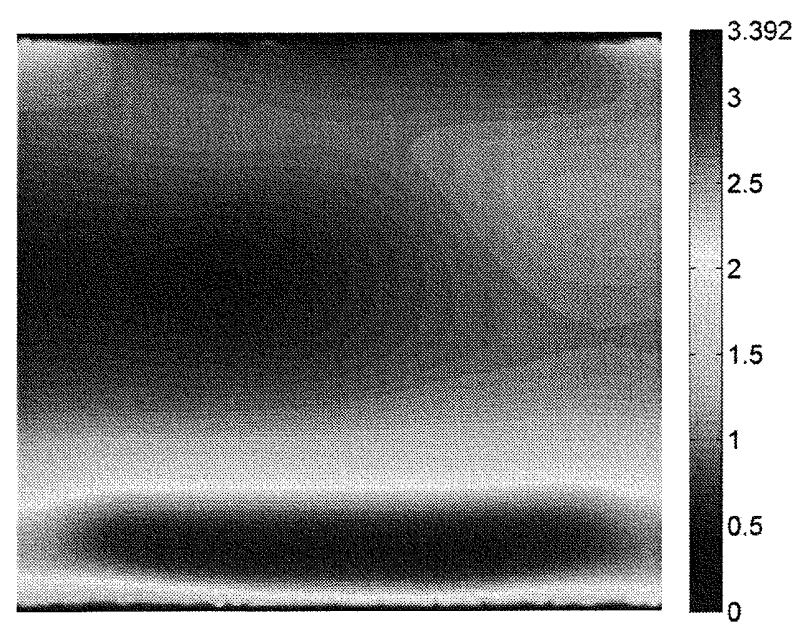

FIG. 2. (Color online) Spatial distribution of percentage error in standard deviation for $\lambda=1$ using seven basis vectors.

lar to the one dealt in [10] - the errors obtained by employing SRBMs are considerably small compared to those obtained using perturbation-based moment methods in that study. Our results also suggest that the errors in the mean and standard deviation tend to decrease when the strength of nonlinearity is increased. This trend can be attributed to the fact that the standard deviation of the response decreases with an increase in the value of $\lambda$ due to the chosen structure for the nonlinearity model. As a consequence, the proposed stochastic Picard iteration scheme converges to improved approximations for the mean and standard deviation of the response.

Figure 2 shows the spatial distribution of the percentage error in standard deviation for the strongly nonlinear case $(\lambda=1)$ when seven basis vectors are employed to approximate the solution process. It can be seen that the maximum error in the standard deviation is $3.4 \%$ which is remarkable considering that we set the variance of the diffusivity field $\sigma_{Y}^{2}=1$.

Theoretically, higher-order PC expansion of basis vectors would lead to improved approximations at the expense of a significant increase in computational complexity. In our numerical studies, we did not find any significant increase in the accuracy of the solutions using higher-order PC representation of the basis vectors. Hence, we have presented the results only with second-order PC expansions which agree well with the benchmark results.

In addition to converging to good approximations, our approach is orders of magnitude faster than the benchmark Monte Carlo simulation when the number PC basis functions is much smaller than the total number of degrees of freedom. To illustrate, consider the number of floating point operations required by MCS and SRBM for a two-dimensional elliptic SPDE spatially discretized on a structured mesh. It can be shown that MCS will take $N_{s} N_{\text {iter }} O\left(n^{2}\right)$ floating point operations, where $N_{s}$ is the sample size, $N_{\text {iter }}$ is the average number of Picard iterations conducted for each realization of the input random variables, and $n$ is the total number of degrees of freedom. On the other hand, SRBMs require $N_{\text {iter }} O\left(n^{1.5}\right)$ to compute the preconditioner at each inexact Picard iteration and an additional $O\left(P P_{u}+P P_{u}^{2}\right)$ matrix-vector operations for Galerkin projection [see Eq. (12)]. In practice if $P, P_{u} \lll n$, it is expected that the computational cost of SRBMs will be orders of magnitude lower than MCS. For the stochastic diffusion problem solved earlier (with $n=2763$ ), SRBMs are about 800 times faster compared to MCS to obtain the first two moments with the same level of accuracy which translates to about 125 deterministic evaluations compared to 100000 evaluations taken by MCS. We expect the computational advantages offered by SRBMs to be more dramatic for problems with more degrees of freedom. However, it is worth noting that when the underlying random field for the diffusivity has a small correlation length, the number of terms in the PC expansion (and random variables arising from discretization) grows rapidly and this would lead to a significant increase in the computational complexity of the proposed approach.

\section{CONCLUDING REMARKS}

In this paper, we propose an inexact Picard iteration scheme for the analysis of nonlinear diffusion processes in random heterogeneous media. The central idea underpinning the proposed formulation is to combine the Picard iteration scheme with generalized stochastic reduced basis methods that employ basis vectors spanning a preconditioned stochastic Krylov subspace. We show the existence of a fixed point for a specific nonlinear stochastic diffusion equation. $\mathrm{Nu}$ merical studies were presented for diffusion in a square domain with stochastic diffusivity. Comparison studies have been conducted for linear and weakly or strongly nonlinear diffusivity models. The results show that the present numerical scheme provides good approximations for the response statistics as the expansion order is increased while taking significantly less computational time compared to standard Monte Carlo simulation.

The proposed approach can be enhanced further by employing adaptive multielement generalizations [29] or by adaptively increasing the number of stochastic basis vectors using estimates of the residual norm. It is also of interest to develop Newton-Raphson-SRBM formulations based on the ideas presented here since this may provide faster convergence rates. The proposed approach is general in scope and can be applied to a wide class of nonlinear stochastic elliptic partial differential equations, for example, deformation analysis of elastoplastic bodies [31] and steady-state fluid flow problems. Finally, a theoretical analysis of the convergence of the proposed iterative numerical scheme would be useful to gain further insights into the convergence characteristics of the stochastic Picard iteration scheme.

\section{ACKNOWLEDGMENTS}

This work was supported by a grant from Rolls-Royce Plc. and the Faculty of Engineering, Science and Mathematics at the University of Southampton. The authors wish to thank Professor A. S. Vasudeva Murthy for valuable discussions on fixed-point theorems. 


\section{APPENDIX}

Proof: To prove that $T(\omega)$ has a fixed point, we use the well-known Leray-Schauder theorem [22]. This theorem states that $T$ has a fixed point if it is a compact mapping from a Banach space $W_{p}^{2} \times L_{p}(\Omega)$ into itself and there exists a constant $M$ such that

$$
\|u\|_{W_{p}^{2} \times L_{p}(\Omega)} \leq M, \quad \forall u \in W_{p}^{2} \times L_{p}(\Omega),
$$

and $\sigma \in[0,1]$ satisfying $u=\sigma T u$.

We now need to show that the operator $T$ defined earlier via the linearized problem (4) is continuous and compact. $T$ maps bounded sets in $W_{p}^{1} \times L_{p}(\Omega)$ into bounded sets in $W_{p}^{2}$ $\times L_{p}(\Omega)$ by the global Schauder estimate [22] which in turn are precompact in $W_{p}^{2} \times L_{p}(\Omega)$ by Arzela's theorem. In order to prove the continuity of $T$, let $\left\{v_{m}\right\}$ be a convergent sequence (converging to $v$ ) in $W_{p}^{1} \times L_{p}(\Omega)$. Then since the sequence $\left\{T v_{m}\right\}$ is precompact in $W_{p}^{2} \times L_{p}(\Omega)$, every subsequence in turn has a convergent subsequence. Let $\left\{T \tilde{v}_{m}\right\}$ be such a convergent subsequence with the limit $u \in W_{p}^{2}$ $\times L_{p}(\Omega)$. Then since

$$
\begin{aligned}
\nabla \cdot & {[\kappa(x, v, \omega) \nabla u(x, \omega)]-f(x, \omega) } \\
= & \lim _{m \rightarrow \infty} \nabla \cdot\left[\kappa\left(x, \bar{v}_{m}, \omega\right) \nabla T \bar{v}_{m}(x, \omega)\right]-f(x, \omega)=0,
\end{aligned}
$$

we must have $u=T v$ and hence the original sequence $\left\{T v_{m}\right\}$ itself converges to $u$. This concludes our proof that a fixed point indeed exists for the map $T$ which gives the solution of the Dirichlet problem given by Eq. (1).
[1] D. Xiu and G. E. Karniadakis, Comput. Methods Appl. Mech. Eng. 191, 4927 (2002).

[2] D. Xiu and G. E. Karniadakis, Int. J. Heat Mass Transfer 46, 4681 (2003).

[3] H. G. Matthies and A. Keese, Comput. Methods Appl. Mech. Eng. 194, 1295 (2005).

[4] A. Keese, Ph.D. thesis, Technische Universitat Braunschweig, Brunswick, 2004.

[5] Eiermann, O. G. Ernst, and E. Ullmann, Comput. Visualization Sci. 10, 3 (2007).

[6] H. Elman and D. Furnival, IMA J. Numer. Anal. 27, 675 (2007).

[7] B. Seynaeve, E. Rosseel, B. Nicolaï, and S. Vandewalle, J. Comput. Phys. 224, 132 (2007).

[8] D. M. Tartakovsky and A. Guadagnini, Phys. Rev. E 64, 035302(R) (2001)

[9] D. M. Tartakovsky, A. Guadagini, and M. Riva, J. Fluid Mech. 492, 47 (2003).

[10] L. Li, H. A. Tchelepi, and D. Zhang, J. Comput. Phys. 188, 296 (2003).

[11] D. Xiu and J. S. Hesthaven, SIAM J. Sci. Comput. (USA) 27, 1118 (2005)

[12] I. Babuška, F. Nobile, and R. Tempone, SIAM (Soc. Ind. Appl. Math.) J. Numer. Anal. 45, 1005 (2007).

[13] G. A. Becus and F. A. Cozzarelli, SIAM J. Appl. Math. 31, 148 (1976).

[14] H. Holden, B. Oksendal, J. Uboe, and T. S. Zhang, Stochastic Partial Differential Equations: A Modelling, White Noise Functional Approach (Birkauser, Boston, 1995).

[15] A. F. Emery and D. Bardot, ASME J. Heat Transfer 129, 1127 (2007).

[16] A. Nouy, Comput. Methods Appl. Mech. Eng. 197, 4718 (2008).

[17] A. Nouy and O. P. Le Maitre, J. Comput. Phys. 228, 202
(2009).

[18] P. B. Nair and A. J. Keane, AIAA J. 40, 1653 (2002).

[19] P. B. Nair, Projection Schemes in Stochastic Finite Element Analysis, Engineering Design and Reliability Handbook, edited by Efstratios Nikolaidis, Dan M. Ghiocel, and Suren Singhal (CRC, Boca Raton, FL, 2005), Chap. 21.

[20] S. K. Sachdeva, P. B. Nair, and A. J. Keane, Comput. Methods Appl. Mech. Eng. 195, 2371 (2006).

[21] M. Renardy and R. C. Rogers, An Introduction to Partial Differential Equations (Springer, New York, 1996).

[22] D. Gilbarg and N. Trudinger, Elliptic Partial Differential Equations of Second Order (Springer-Verlag, Berlin, 1977).

[23] R. Ghanem and P. Spanos, Stochastic Finite Elements: A Spectral Approach (Springer, New York, 1991).

[24] K. E. Atkinson, The Numerical Solution of Integral Equations of the Second Kind (Cambridge University Press, Cambridge, England, 1997).

[25] R. G. Ghanem, ASME J. Appl. Mech. 66, 964 (1999).

[26] J. A. S. Witteveen and H. Bijl, in Proceedings of the 44th AIAA Aerospace Sciences Meeting and Exhibit (American Institute of Aeronautics and Astronautics, Reno, NV, 2006), p. 20060896.

[27] P. B. Nair, in Proceedings of 42nd AIAA/ASME/ASCE/AHS/ ASC Structures, Structural Dynamics and Materials Conference, AIAA Paper No. 2001-1677 (American Institute of Aeronautics and Astronautics, Seattle, WA, 2001).

[28] S. K. Sachdeva, P. B. Nair, and A. J. Keane, Probab. Eng. Mech. 21, 182 (2006).

[29] P. S. Mohan, P. B. Nair, and A. J. Keane, Comput. Methods Appl. Mech. Eng. 197, 1495 (2008).

[30] R. H. Cameron and W. T. Martin, Ann. Math. 48, 385 (1947).

[31] M. Anders and M. Hori, Int. J. Numer. Methods Eng. 51, 449 (2001). 
